\title{
Identidad personal y compromiso deportivo en adolescentes nadadoras de nivel competitivo
}

\section{Personal identity and sports commitment in adolescent swimmers of competitive level}

\author{
Bárbara Hernández y Claudia Capella \\ Universidad de Chile, Santiago, Chile
}

\section{Resumen}

La presente investigación tiene como objetivo comprender la forma en que las adolescentes nadadoras de nivel competitivo construyen su identidad personal y cómo esto se relaciona con la construcción de significados asociados al compromiso deportivo. Se utilizó una metodología de carácter cualitativo, con un enfoque constructivista evolutivo, con el objetivo de acceder a los significados construidos por las propias adolescentes con respecto a la práctica deportiva y al lugar que ocupa en su construcción de identidad. Se recurrió al uso de autobiografías escritas y entrevistas semiestructuradas, las que posteriormente fueron sometidas a análisis de contenido. A partir de los resultados, se concluye que la práctica deportiva de natación a nivel competitivo puede ocupar tres lugares en la construcción de identidad adolescente, los cuales se relacionan con un mayor o menor compromiso deportivo. Además, se dio a conocer la construcción de significados asociados a la práctica deportiva, que se vincularían a una mayor permanencia en el deporte o a su abandono. Finalmente, se enfatizaron los aportes que desde una mirada constructivista evolutiva se pueden realizar a la investigación e intervención en Psicología Deportiva, al considerar no solo los aspectos competitivos, sino también el logro de un desarrollo adaptativo.

Palabras clave: identidad, adolescencia, Psicología Deportiva, nadadoras, constructivismo evolutivo.

\begin{abstract}
The aim of this research is to understand how adolescent swimmers of a competitive level build their personal identity and how this relates to the construction of meanings associated with sport commitment. A qualitative methodology was used, from an evolutive constructivist approach, in order to access to the meanings constructed by the adolescents about sport and the place it has in the construction of identity. Autobiographies and semi-structured interviews were used, which then were subjected to content analysis. From results, it was concluded that, the practice of competitive swimming can have three places in the construction of adolescent identity. These places are related to a greater or lesser sports commitment. It was also disclosed the construction of meanings associated with sports practice that would bind greater permanence in sport or abandonment. Finally, the contributions from a constructivist evolutive perspective in sports psychology research are emphasized, to consider not only the competitive aspect but also the achievement of a comprehensive and adaptive development.
\end{abstract}

Keywords: identity, adolescence, sports psychology, swimmers, evolutive constructivism.

Contacto: C. Capella. Av. Ignacio Carrera Pinto 1045, Nuñoa, Santiago, Chile. ccapella@u.uchile.cl

Cómo citar este artículo:

Hernández, B. y Capella, C. (2014). Identidad personal y compromiso deportivo en adolescentes nadadoras de nivel competitivo. Revista de Psicología, 23(1), 71-83. doi: 10.5354/0719-0581.2014.32875 


\section{Introducción}

Un aspecto relevante en relación al deporte de rendimiento es la permanencia y abandono deportivo a edades tempranas del desarrollo (Vázquez, 2008). Investigaciones realizadas en diversos contextos y deportes destacan que entre los 12 y 18 años existe un marcado índice de abandono deportivo (Guillén, Weis y Navarro, 2005; Haro Navejas, 2007), con preponderancia en las adolescentes mujeres (Díez y Hernández, 2006).

La permanencia deportiva se ha relacionado con lo definido como compromiso deportivo, que sería una disposición psicológica vinculada al deseo y decisión de seguir participando en el deporte (Torregrosa et al., 2007). Autores como Haro Navejas (2007), Sparkes y Devis (2007), Sparkes y Smith (2001), plantean la importancia del constructo de identidad deportiva, definido como "el grado en que las personas se identifican con el rol de deportista" (Brock y Kleiber 1994, en Vázquez, 2008, p. 3), el cual se va elaborando a través de las distintas etapas del desarrollo del sujeto y se relaciona con el mantenimiento de la práctica deportiva y la percepción que cada deportista posee respecto a su capacidad dentro del deporte que practica. Se ha planteado la importancia de la identidad deportiva con una mayor participación en el deporte, tanto recreativo como competitivo (Anderson, Masse, Zhang, Coleman, Chang, 2009; Wylleman y Lavalle, 2004). De este modo, la construcción de identidad a través del deporte se podría relacionar con un mayor compromiso hacia la práctica deportiva.

Junto con esto, estudios han mostrado que en el abandono deportivo influirían aspectos como: metas elevadas, ambiente negativo con los compañeros de equipo, falta de comunicación con el entrenador, débil apoyo social, baja percepción de realización personal; siendo los factores más influyentes en el abandono de la práctica deportiva, el que esta se focalice exclusivamente en los resultados obtenidos y en la percepción de una excesiva monotonía y demanda temporal (Cudini, 2003; Garcés, 1995).

Dado lo anterior, sería posible caracterizar a un gran número de deportistas adolescentes que pueden llegar a alejarse de una práctica activa, a la que consideran altamente desgastante (Vasalo, 2001). Aunque autores como Ruiz, Durán y Arruza (2007) plantean que luego de miles de horas de práctica se llega a una satisfacción corporal relacionada con un nivel de exigencia física y cognitiva que en los deportistas de nivel competitivo se constituye como una fuente de satisfacción, derivada del ser y sentirse competentes.
Tal como se señaló anteriormente, existiría un rango etario en donde es posible identificar el mayor índice de abandono deportivo de nivel competitivo, que abarca una parte importante de lo que la Organización Mundial de la Salud (OMS, 2011) considera como adolescencia, definiéndole como el periodo evolutivo que va desde los 10 a los 19 años de edad.

Desde el enfoque evolutivo, se plantea la relevancia de considerar las características del período del desarrollo de los sujetos (Sepúlveda, 2008), por lo que cabe preguntarse si las tareas evolutivas propias de la adolescencia podrían influir en el alto abandono deportivo en esta etapa. En este sentido y considerando los planteamientos de la perspectiva constructivista evolutiva, se destaca la adolescencia como una importante etapa de formación, en donde la meta central es la construcción de la identidad personal, que se caracterizaría por un proceso de diferenciación e integración del sí mismo, que implica necesariamente el reconocimiento e integración de los otros (Sepúlveda, 2006).

De tal modo, se plantea el término identidad personal como la estructura del sí mismo que la persona construye internamente, caracterizada por tres procesos:la organización de la unidad del sí mismo, la integración de sí mismo y la integración con otros (Sepúlveda, 2008). El primero de ellos hace referencia a la afirmación del sí mismo y a la necesidad de un reconocimiento de sí como ser único y diferente, con características propias. Mientras que la integración del sí mismo se relaciona con la acomodación a experiencias que se han dado en el transcurso de la historia vital, integrando pasado, presente y futuro. Finalmente, mediante el concepto de integración con otros se alude al reconocimiento de los otros como parte de sí y la diferenciación con estos (Sepúlveda, 2006).

La presente investigación considera estos aportes al momento de estudiar problemáticas surgidas desde el ámbito deportivo, en este caso, el compromiso deportivo. Desde la perspectiva constructivista evolutiva que guiará este estudio, surge la interrogante de si, debido a la importancia de la construcción de identidad en la adolescencia, puede existir un vínculo entre compromiso deportivo e identidad personal.

Se utilizará una perspectiva cualitativa que integre aspectos constructivista evolutivos en el abordaje de problemáticas referidas al deporte, ya que se pretende abordar la construcción de identidad y el modo en que las personas son capaces de significar sus experiencias, accediendo así a los significados que los propios deportistas atribuyen a la actividad que practican, realizando descripciones desde 
las categorías que ellos mismos utilizan. Se destaca además que los métodos cualitativos, son cada vez más frecuentes en el estudio de las problemáticas asociadas al deporte y la actividad física (Sparkes y Devis, 2007).

En lo referente a la natación competitiva en Chile, existen alrededor de 700 nadadores inscritos en la Federación Chilena de Deportes Acuáticos (Fechida), dentro de ellos, más de las tres cuartas partes son nadadores adolescentes, y sólo alrededor de 10 tienen financiamiento estatal o privado (Carbonell, 2008). Considerando este contexto, que gran parte del abandono deportivo ocurre en adolescentes mujeres (Díez y Hernández, 2006) y ante la escasez de investigaciones referidas al compromiso deportivo en deportistas adolescentes chilenas, la pregunta que guió la presente investigación fue:

¿Cómo es la construcción de identidad personal en nadadoras adolescentes de nivel competitivo y cómo se relaciona con los significados asociados al compromiso deportivo?

Para responder esta pregunta, el objetivo del estudio fue comprender la forma en que las adolescentes nadadoras de nivel competitivo construyen su identidad y su relación con la construcción de significados asociados al compromiso deportivo. Para ello se recurrió al uso de entrevistas y autobiografías realizadas a 10 adolescentes de sexo femenino dedicadas a la práctica deportiva de natación competitiva.

\section{Método}

\section{Participantes}

La muestra de la presente investigación es de carácter intencionado, no probabilístico, en donde participaron 10 nadadoras adolescentes de nivel competitivo cuyas edades fluctuaban entre los 11 y 19 años. Todas pertenecían a un club deportivo de la ciudad de Santiago de Chile, que se caracteriza por su extensa trayectoria en la disciplina de natación, al brindar cursos de formación y poseer una de las ramas de natación competitiva más antiguas del país. $\mathrm{Al}$ ser un muestreo teórico de características intencionadas, se estableció como criterio de selección que las participantes tuviesen edades dentro del rango definido como adolescencia por la OMS (2011). Se procuró además que dentro del colectivo se distinguiera la presencia de adolescentes de diversas edades, que hubieran dejado en algún momento la práctica deportiva y otras que se caracterizaran por su constancia y destacada trayectoria competitiva. De este modo se buscaba que la muestra fuese lo más heterogénea posible dentro de su homogeneidad.
Dentro de los criterios considerados como relevantes para la selección, destaca además el tiempo que las adolescentes le dedicaban a la práctica deportiva de natación. Para que esta fuese considerada de nivel competitivo tiene que existir una dedicación diaria, la que según Carbonell (2008) va desde un mínimo de dos horas diarias en al menos cinco días a la semana. Además es necesario que las adolescentes estuviesen inscritas en la Fechida y que hubiesen participado en competencias de nivel federado. Todos estos aspectos mencionados dan cuenta del nivel competitivo en que está inserta la muestra.

Las características de las participantes son descritas en la tabla 1.

Cabe señalar que al momento de las entrevistas todas las participantes asistían de forma regular a los entrenamientos, salvo las participantes 4 y 8 , que asistían de manera irregular.

\section{Materiales}

Se recurrió al uso de autobiografías escritas y entrevistas semiestructuradas, las que fueron usadas con el mismo fin y de modo complementario para indagar en la percepción que las adolescentes tenían respecto a las temáticas de la investigación (Capella, 2013). El uso de ambas técnicas favoreció la triangulación, en la medida que permitió integrar diferentes miradas del fenómeno a investigar (Cornejo y Salas, 2011).

En cuanto a las autobiografías, son una técnica característica de la evaluación constructivista, que permite acceder a la construcción y significación que la persona realiza, tanto de sí mismo y su historia vital, como de las relaciones que establece. Esto favorece el estudio de aspectos relacionados con el sí mismo y la identidad (Berntsen y Rubin, 2006, en Capella, 2011).

El formato de la autobiografía utilizado corresponde al planteado por Sepúlveda con adaptaciones de Capella (2011), que presenta la siguiente consigna a los adolescentes: "Escribe una historia sobre ti y tu vida, pudiendo incluir aspectos tales como por ejemplo, una presentación personal en cuanto a quién eres, cuáles han sido los hechos o situaciones más importantes de tu vida, cómo imaginas tu futuro, entre otros".

En cuanto a las entrevistas semiestructuradas, en ellas el entrevistador desea obtener información sobre cierto problema a investigar y a partir de este, se establece una lista de tópicos o temas a abordar (García, Gil y Rodríguez, 1999). En este caso puntual se retomaron los 
Tabla 1

Caracterización de la muestra

\begin{tabular}{|c|c|c|c|}
\hline Entrevistada & Edad & $\begin{array}{l}\text { Años de dedicación a } \\
\text { la práctica de forma } \\
\text { competitiva }\end{array}$ & Experiencia competitiva en natación \\
\hline 1 & 16 años & 4 años & Participación en competencias nacionales \\
\hline 2 & 14 años & 2 años & Participación en competencias nacionales \\
\hline 3 & 17 años & 10 años & $\begin{array}{c}\text { Participación en competencias nacionales } \\
\text { e internacionales a nivel sudamericano. } \\
\text { Fue seleccionada nacional. }\end{array}$ \\
\hline 4 & 16 años & 6 años & Participación en competencias nacionales \\
\hline 5 & 16 años & 6 años & $\begin{array}{c}\text { Participación en competencias nacionales } \\
\text { e internacionales a nivel sudamericano. } \\
\text { Es seleccionada nacional. }\end{array}$ \\
\hline 6 & 18 años & 4 años & Participación en competencias nacionales \\
\hline 7 & 13 años & 7 años & $\begin{array}{c}\text { Participación en competencias nacionales } \\
\text { e internacionales a nivel sudamericano. } \\
\text { Es seleccionada nacional. }\end{array}$ \\
\hline 8 & 16 años & 7 años & Participación en competencias nacionales \\
\hline 9 & 11 años & 3 años & Participación en competencias nacionales \\
\hline 10 & 12 años & 4 años & Participación en competencias nacionales \\
\hline
\end{tabular}

aspectos más relevantes presentes en las autobiografías, profundizando en aquellos temas relacionados con los objetivos de la investigación que no habían sido mencionados explícitamente con anterioridad. Se destaca como principales tópicos de la entrevista (ver tabla 2) aquellos relacionados con la visión que las adolescentes tienen de sí, como también aspectos vinculados a los significados de la práctica deportiva.

\section{Procedimiento}

Inicialmente se solicitó y obtuvo la autorización del encargado de los entrenamientos en un club de natación en Santiago de Chile. Luego se informó a los padres y adolescentes de las características de la investigación y se les invitó a participar. A quienes accedieron, se les solicitó la firma de un documento de consentimiento informado a los padres y un asentimiento informado a las adolescentes.

Luego de esto, se realizaron las autobiografías y entrevistas, de manera individual, durante septiembre del año 2011, en una sala privada dentro del recinto de entrenamiento de las adolescentes. Primero, se les extendió la consigna para la autobiografía, dándoles el tiempo que requiriesen, para posteriormente realizar la entrevista en base a la pauta semiestructurada y retomando ciertos aspectos planteados en la autobiografía. El procedimiento de escritura de la autobiografía y posterior realización de la entrevista fue similar en todas las participantes, no observándose variaciones relevantes de acuerdo a la edad, aun cuando la aplicación se adecuó a las características de cada una.

Tabla 2

Tópicos de las entrevistas semiestructuradas

\begin{tabular}{ll}
\hline Aspectos de sí mismos & Significados de la práctica deportiva \\
\hline Características de sí mismas & Inicios en la natación \\
Actividades que les gusta realizar & Significados de la natación \\
Experiencias relevantes & Aspectos que consideren más relevantes de la práctica y su permanencia en esta \\
Visión de su vida a futuro & Dificultades presentes en la práctica deportiva \\
Relaciones con la familia & Metas y objetivos asociados al deporte \\
$\begin{array}{l}\text { Amigos y relación con el grupo de } \\
\text { pares }\end{array}$ & Caracterización de la relación con el entrenador y compañeros de práctica \\
\hline
\end{tabular}


Las narrativas autobiográficas escritas por las adolescentes tuvieron un promedio de 635 palabras. En cuanto a las entrevistas, estas fueron realizadas en un tiempo promedio de 76 minutos.

El método utilizado para el análisis de la información fue el análisis de contenido, con un criterio de clasificación de categorías temáticas (Andréu, 2000). Inicialmente se realizó un análisis de cada autobiografía y cada entrevista, identificando temas centrales, para luego organizarlos de acuerdo a ámbitos temáticos (Cornejo, Mendoza y Rojas, 2008). El análisis fue realizado por separado, y luego de forma integrada, considerando tanto autobiografías como entrevistas de cada caso. Posteriormente, se realizó un análisis observando similitudes y diferencias entre casos, realizándose con ello una triangulación de la información obtenida (Capella, 2013).

Se integraron las distintas categorías de contenido, clasificándolas y ordenándolas, aunando las principales temáticas surgidas. Estas se organizan en torno a ejes centrales, en diferentes niveles de integración, dando coherencia a los datos obtenidos en función de los objetivos de la investigación. Las categorías trabajadas surgieron del análisis, realizado de forma manual caso a caso, no encontrándose estas definidas con anterioridad. Se enfatiza en que el análisis de contenido fue revisado en equipo, lo que permitió su triangulación y validez (Cornejo y Salas, 2011).

\section{Resultados}

Para comprender la forma en que las adolescentes nadadoras de nivel competitivo construyen su identidad y la relación de esta con la construcción de significados asociados al compromiso deportivo, los resultados de la presente investigación se presentarán organizados en dos ejes, el primero referido a la identidad y las diferentes posiciones de la práctica deportiva en esta. En segundo lugar, expondremos los resultados respecto a los factores que las adolescentes identifican vinculados al compromiso deportivo, ligados por ellas al abandono y permanencia deportiva. En ambos apartados se presentarán fragmentos representativos de las entrevistas y autobiografías ${ }^{1}$.

1 Lo que aparece entre comillas son citas textuales extraídas de las entrevistas o autobiografías. Las participantes fueron numeradas al azar (numeración referida en la tabla 1), por lo cual luego de cada cita se explicita la participante. La E. corresponde a una viñeta de la entrevista y la A. a una viñeta de la autobiografía. (Ejemplo: A.9 se refiere a autobiografía de la participante 9; E.1 a entrevista de la participante 1).
Identidad y posiciones de la práctica deportiva en la identidad

A continuación se presentan los aspectos más destacados por las participantes en torno a la identidad, organizados de acuerdo a las tres dimensiones de la identidad personal que fueron observadas en las narrativas: unidad del sí mismo, integración del sí mismo e integración con otros. Se destacarán los aspectos comunes observados en las participantes así como sus diferencias.

Unidad del sí mismo. En la caracterización personal que las adolescentes realizan de sí mismas, la práctica deportiva surge frecuentemente como un elemento constituyente de la visión de sí mismas. Sin embargo, se observan diferencias. En algunos casos el "ser" nadadora se posiciona como elemento central en la autodefinición, o las características personales identificadas como relevantes se vinculan a la práctica deportiva como, por ejemplo, ser competitivas.

"Mi nombre es Constanza² (...) Tengo 12 años, soy nadadora”. A. 9.

"Soy muy competitiva conmigo misma, me gusta estar siempre así, así como mejorando (...) sobre todo por esto de la natación”. E. 1.

En otras adolescentes, aun cuando la caracterización personal integra aspectos relacionados a la práctica deportiva, incluyen también otras valoraciones, vinculadas a su forma de ser, que se relacionan no necesariamente con el deporte.

"Soy Macarena, tengo 15 años de edad, voy en segundo medio y practico natación. (...) Soy una persona un poco cuadrada en cuanto a la forma de pensar, simpática cuando quiero y ando de buen humor. Me considero una persona feliz, con una familia que me apoya, no soy de esas personas que se preocupan mucho de otras, no soy de salir mucho y tampoco soy muy conversadora. Me gusta y disfruto mucho lo que hago". A. 5.

En algunos casos, la práctica deportiva no constituye un elemento central respecto a la visión de sí mismas, describiéndose algunas adolescentes en torno a otras características o intereses no ligados al deporte (e.g., intereses escolares).

2 Todos los nombres o cualquier otra referencia explícita a los participantes de esta investigación han sido cambiados y/o omitidos en pos de resguardar su identidad. 
Integración del sí mismo. Respecto a la integración de sí mismo, se aprecian dos elementos fundamentales, uno relacionado a los hechos vitales significativos, y otro referido a la visión de futuro. En el primero de ellos, las adolescentes destacan como hechos significativos en sus vidas aquellos relacionados a la práctica deportiva (inicios en la natación, entrenamientos, primera competencia).

En algunos casos, los eventos significativos descritos se vinculan casi exclusivamente a la práctica deportiva.

"Entrar a la Rama, porque estos tres años han sido los mejores de mi vida (...). Desde chica ya pensaba en meterme a la Rama, era como mi meta, ahora mi meta ya no es entrar a la Rama, sino ser una nadadora profesional". E. 9.

Otras adolescentes, junto con hechos vinculados a la natación, incluyen también en el relato, la descripción de otros eventos vitales significativos, como cambiar de colegio, emigrar a otro país/ciudad, nacimiento de hermanos, pololear, entre otros.

"Uno de los hechos importantes fue empezar mi pololeo, ha sido una de las cosas más importantes en mi vida (...). El otro hecho importante fue comenzar la natación, porque fue algo que definitivamente cambió mi vida”. A. 5.

En otros casos, los eventos relevantes señalados no guardan relación con la práctica deportiva, pero esta es considerada en ocasiones como un elemento que da continuidad a la historia vital.

"Llegué a Chile hace 4 años y medio, fue uno de los más grandes cambios en mi vida, porque el estilo de vida en Colombia es mucho más agitado que en Chile”. A. 6.

"Todos esos hechos importantes de los que ya hablé pasaron cuando ya estaba acá, ya nadaba, entonces como que yo todo eso siempre lo he vivido acá". E. 8.

Respecto a la visión de futuro, esta se centra principalmente en proyecciones deportivas y/o académicas/profesionales.

En algunos casos, prima la visión de futuro centrada en las proyecciones deportivas, manifestándose deseos de mantenerse en la práctica de forma constante, mejorando las propias marcas. El proyectar continuidad en la práctica deportiva se puede asociar a que esta se constituye como un elemento central en sus vidas en el presente y futuro.
"No me imagino no hacer natación, o sea, sin la piscina, no entrenando (...) dejarlo no, que siento que es como una parte de mi vida, como no podría dejarlo botado así por cualquier cosa”. E. 1 .

“Ser buena deportista, no sé, ojalá llegar a ser la mejor, la más importante (...) dedicarme, llegar así como la Kristel Köbrich ${ }^{3}$, dedicarme esto al máximo y nadar, nadar, nadar". E. 7.

En otros casos, se visualiza una continuidad en la práctica deportiva a futuro, pero complementaria con otros aspectos, como los académicos, profesionales y familiares, concibiendo la capacidad de compatibilizar diversas actividades.

"Yo me veo estudiando y me imagino también practicando natación, porque es lo que más me gusta (...) y en la piscina como mejorando mis marcas siempre". E. 2.

"Me veo siempre nadando, aunque esté estudiando. Igual me gustaría meterme a un preu, pero que sea los días sábados, así no tendría que faltar a entrenar ni nada (...), y no sé, después tener una familia, que me vaya bien, así tener hartos hijos, como 3 ó 4 y a todos meterlos a un deporte, al que ellos les guste, pero a un deporte." E. 5.

Excepcionalmente, en otros casos, se manifiesta inseguridad respecto a la continuidad en la práctica deportiva, aunque se reconocen proyecciones académicas y/o profesionales. Esta visión se observa en aquellas nadadoras cuya práctica ha sido más inconstante.

"Yo creo que voy a seguir por lo menos hasta que termine el colegio, de ahí no sé (...), tal vez si entro a la (Universidad de) Chile siga nadando, es que lo encuentro bonito, porque es como todo un proceso, porque he pasado, como te dije, los momentos más importantes acá, entonces igual me gustaría que siguiese así”. E. 8 .

Integración con los otros. En cuanto a la visión de los otros, sobresale en las adolescentes una visión positiva de los otros cercanos, describiendo frecuentemente vínculos positivos con ambos padres, hermanos, familiares próximos, los amigos y la figura del entrenador. Las figuras parentales son integradas como parte relevante en cuanto a lo que las adolescentes son, mencionando situaciones que ejemplifican

3 Destacada nadadora chilena de trayectoria a nivel nacional e internacional. 
cuán importante es el apoyo percibido en el ámbito deportivo y en otras áreas de su vida.

En cuanto al grupo de pares, las adolescentes diferencian claramente entre "amistades del colegio" y "de natación". En algunas adolescentes, el grupo de pertenencia de pares corresponde principalmente a aquellos vínculos presentes al interior del equipo de natación.

"Yo siento que me dedico más a mis amigos de natación". E. 7.

En otras adolescentes existe una integración de distintos vínculos afectivos con pares, aun cuando se describe que es el grupo de amigos de natación con los que se siente una mayor pertenencia, al compartir las mismas preferencias e intereses.

"Mis amigos de acá son más cercanos que los del colegio, les veo todos los días (...) compartimos lo mismo que nos gusta (...) Con los amigos del colegio no compartimos lo mismo así, porque ellos no entienden lo que es hacer un deporte, en cambio los de la piscina entienden eso y como que también quieren mejorar (...) ellos son más cercanos”. E. 2.

Excepcionalmente hay casos en que el grupo de pertenencia de pares significativos no se encuentra presente en la práctica deportiva, sino que en el colegio. Esta visión coincide con adolescentes que han permanecido de forma inconstante en la práctica deportiva.

"Mis amigas de colegio son como que son súper parecidas a mí, la misma música, los gustos, y nos conocemos, compartimos mucho". E. 8.

En cuanto a los aspectos diferenciales observados en las tres dimensiones de la identidad, desde el análisis realizado se desprende que la práctica deportiva se puede posicionar de tres modos distintos en relación a la construcción de la iden-

Tabla 3

Posiciones de la práctica deportiva en la identidad personal adolescente

\begin{tabular}{|c|c|c|c|}
\hline $\begin{array}{l}\text { Dimensiones de la iden- } \\
\text { tidad personal }\end{array}$ & $\begin{array}{l}\text { Identidad centrada en la } \\
\text { práctica deportiva }\end{array}$ & $\begin{array}{l}\text { Identidad integradora de la práctica } \\
\text { deportiva }\end{array}$ & $\begin{array}{l}\text { Identidad no centrada } \\
\text { en la práctica }\end{array}$ \\
\hline Unidad del sí mismo & $\begin{array}{l}\text { El “ser" nadadora se po- } \\
\text { siciona como elemento } \\
\text { central en cuanto a la de- } \\
\text { finición de sí mismas. }\end{array}$ & $\begin{array}{l}\text { La caracterización personal integra } \\
\text { aspectos relacionados a la práctica } \\
\text { deportiva con otras valoraciones. }\end{array}$ & $\begin{array}{l}\text { La práctica no constitu- } \\
\text { ye un elemento central } \\
\text { respecto a la visión de sí } \\
\text { mismas. }\end{array}$ \\
\hline \multirow[t]{2}{*}{ Integración del sí mismo } & $\begin{array}{l}\text { Se hace referencia a even- } \\
\text { tos significativos asocia- } \\
\text { dos principalmente a la } \\
\text { práctica deportiva. }\end{array}$ & $\begin{array}{l}\text { Se relacionan eventos vitales vincu- } \\
\text { lados tanto a la práctica deportiva } \\
\text { como a otras vivencias. }\end{array}$ & $\begin{array}{l}\text { Los principales eventos } \\
\text { centrales señalados no } \\
\text { guardan relación con la } \\
\text { práctica deportiva, pero } \\
\text { esta es considerada en } \\
\text { ocasiones como un ele- } \\
\text { mento que da continui- } \\
\text { dad a la historia vital. }\end{array}$ \\
\hline & $\begin{array}{l}\text { Visión de futuro centrada } \\
\text { principalmente en pro- } \\
\text { yecciones deportivas. }\end{array}$ & $\begin{array}{l}\text { Se visualiza una continuidad en la } \\
\text { práctica deportiva a futuro, con inte- } \\
\text { gración de otros aspectos, como los } \\
\text { académicos o profesionales. }\end{array}$ & $\begin{array}{l}\text { A futuro, se manifiesta } \\
\text { inseguridad respecto a la } \\
\text { continuidad en la prác- } \\
\text { tica deportiva, pero se } \\
\text { reconocen proyecciones } \\
\text { académicas y/o profesio- } \\
\text { nales. }\end{array}$ \\
\hline Integración con los otros & $\begin{array}{l}\text { El grupo de pertenencia de } \\
\text { pares corresponde primor- } \\
\text { dialmente a aquellos vín- } \\
\text { culos presentes al interior } \\
\text { del equipo de natación. }\end{array}$ & $\begin{array}{l}\text { Existe una integración de distintos } \\
\text { vínculos afectivos con pares. }\end{array}$ & $\begin{array}{l}\text { El grupo de pertenencia } \\
\text { de pares significativos no } \\
\text { se encuentra presente en } \\
\text { la práctica deportiva. }\end{array}$ \\
\hline
\end{tabular}


tidad personal adolescente, identificándose que cada una de las nadadoras se ubica de manera preferente en uno de estos diferentes modos: identidad centrada en la práctica deportiva (participantes $1,7,9$ ); identidad integradora de la práctica deportiva (participantes 2, 3, 5); e identidad no centrada en la práctica (participantes $4,6,8,10$ ). En la tabla 3 se resumen sus principales características, descritas previamente, considerando las tres dimensiones de la identidad.

La identidad centrada en la práctica e integradora de la práctica se presentaban en adolescentes que podía identificarse que en sus relatos poseían un mayor compromiso deportivo, en tanto que la identidad no centrada en la práctica se relacionaría con adolescentes que presentaban en sus relatos una descripción de la práctica de manera más inconstante, por lo cual, podríamos suponer, con un mayor riesgo de abandono deportivo.
Aspectos asociados al abandono y la permanencia deportiva

Junto con la descripción de la identidad, se enfatizó en aquellos aspectos relacionados con el ámbito deportivo, como el compromiso deportivo y los significados asociados a la práctica. Desde el análisis realizado, es posible identificar en las narrativas de las adolescentes, significados del compromiso deportivo, en torno a dos ejes centrales. Por una parte se reconocen aquellos aspectos considerados positivos por las participantes y que incidirían en la permanencia en la práctica deportiva de rendimiento. En el segundo eje en cambio, las adolescentes describen las principales dificultades, que podrían asociarse al abandono deportivo. A continuación, y desde el análisis de las narrativas, se da cuenta de estos elementos, que se resumen en la figura 1 y luego se describen, incluyendo viñetas de las narrativas de las participantes.

Figura 1

Significados atribuidos al compromiso deportivo

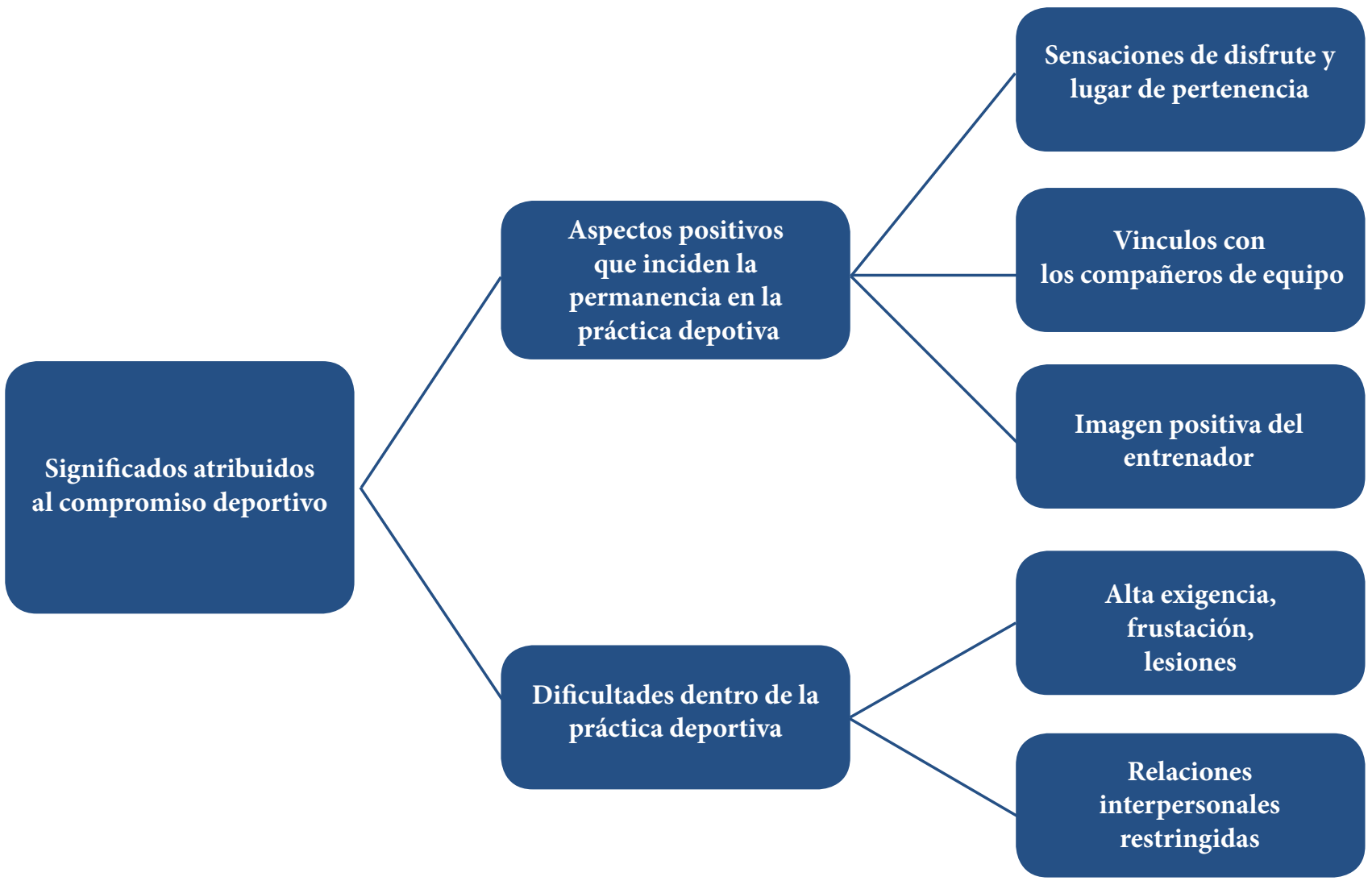


Sensaciones de disfrute y lugar de pertenencia. En cuanto a la construcción de significados vinculados a la natación, estos aparecen de manera espontánea en los relatos de las adolescentes, vinculados a sus deseos de permanencia en el deporte. Es frecuente que relacionen el espacio que ocupa la práctica deportiva con un lugar de tranquilidad y disfrute, en donde las responsabilidades académicas y otras preocupaciones son olvidadas durante el tiempo que dura el entrenamiento, lo que facilita que este espacio se constituya como un lugar de pertenencia y satisfacción personal.

"Es como un escape a todas las otras cosas, del estrés del colegio, de la casa, de todo (...) como que es una parte importante ya de la vida, es como mi lugar”. E. 6.

"Es como entretenido, te despeja, se pasa bien sí o sí (...) La natación es otra cosa, es como perfecta”. E. 1.

"Me encanta el agua, ehhh, me relajo nadando, no sé, me hace feliz". E. 4.

Vínculo con los compañeros de equipo. Por otro lado, la relación positiva con los amigos del equipo de natación es frecuentemente descrita por las nadadoras como relevante, siendo para ellas un factor decisivo tanto al momento de comenzar la práctica deportiva como al mantenerse en ella.

"Cuando vi el ambiente, la gente y cómo lo pasaban, eso como que me enganchó, eso fue como lo que me atrajo a seguir, a la natación, luego bajar mis tiempos y seguir (...) Te entretení con la gente que está acá, la pasái bien, entonces como que te vuelves a motivar, si hay buen ambiente obvio que te va a gustar llegar temprano, venir a conversar, reírte cuando parái en los descansos, igual como que te ayuda todo ese ambiente". E. 5.

Imagen positiva del entrenador. Respecto al entrenador, las adolescentes lo mencionan espontáneamente como una figura significativa en sus vidas, caracterizándola positivamente como una persona cercana. En algunos casos, incluso es vinculado a una imagen paterna.

"Una persona que encuentro que es muy especial para mí es mi entrenador". A. 7.

"Como la confianza que se va formando con él es como la de un papá o algo así, no es como simplemente tu entrenador, al final es como parte de tu familia”. E. 2.
El que la figura del entrenador ocupe un lugar privilegiado dentro de los vínculos significativos que describen las adolescentes no significa que la relación esté exenta de ciertas dificultades. Sin embargo, se destaca como una persona cariñosa, confiable, atenta a que mejoren sus rendimientos, lo que incide en su permanencia.

"Yo lo encuentro muy especial, a pesar de que me rete, pero porque me exige más, yo encuentro que es porque me quiere, porque quiere que lo haga bien”. E. 7 .

"Como que entiende todo lo que decí, antes de hablar ya sabe todo lo que le quieres contar, como que me encanta, como es como persona, como hace su trabajo, es muy profesional, me agrada estar con él aparte está todas las tardes (...) es agradable estar aquí con él (...) él influye mucho, porque depende de los dos los entrenamientos". E. 3.

"Yo no sé si hubiese otro entrenador si seguiría viniendo". E. 8 .

Alta exigencia, frustración, lesiones. Surge también en las adolescentes descripciones de sensaciones como angustia o frustración, relacionadas con exigencia y sensación de no estar rindiendo como ellas o su entrenador espera que lo hagan, lo cual se relaciona desde sus discursos con deseos de abandonar el entrenamiento.

"De repente en entrenamiento, yo siento que no puedo, no puedo, siento que me angustia no puedo, como que no avanzo nada (...) y como que pienso que me voy a salir del agua, me desmotivo". E. 7.

Otra de las frustraciones descritas es el hecho de no bajar las marcas -relacionado a mejor rendimiento-, pese a la constancia en los entrenamientos. La solución para la mayoría de las adolescentes consiste en mantenerse en la rutina hasta volver a rendir.

"De repente cuando a uno le va mal, se frustra, pero es lo mínimo, porque uno después piensa que pa' la otra lo haré mejor". E. 10.

Excepcionalmente, surge en el relato de dos adolescentes temáticas relacionadas a las lesiones deportivas, que se asocian a sensaciones de frustración y angustia.

"Es que no podía seguir entrenando, me dolía mucho el hombro, había tenido también una lesión antes en los 
muslos y tendinitis, entonces no podía bracear, no podía patear y nada (...) Estuve quince días en cama, llorando a mares, no tenía ánimo de nada, tenía un brazo menos, fue lo peor que me ha pasado en la vida, nunca más quiero pasar por eso (...) Necesitaba volver a meterme en una piscina y así fue cuando volví el 1 agosto del 2011 a entrenar en la piscina de toda mi vida”. E. 3 .

Relaciones interpersonales restringidas. Otra de las problemáticas vinculadas por las adolescentes a la posibilidad de abandono se relaciona con los vínculos interpersonales, que podrían verse limitados solo al espacio de la práctica deportiva, alejándoles de otros intereses propios de la etapa evolutiva en que se encuentran (e.g.,. asistir a fiestas o encuentros con pares). Así, identifican dificultades en establecer vínculos o participar en actividades con pares fuera del ambiente deportivo, principalmente debido a la gran cantidad de horas que dedican a la práctica deportiva.

"Ahí yo sentía que casi el venir a nadar me quitaba tiempo para hacer otras cosas... No sé, salir a casa de mis amigas, porque mis amigas decían: 'pero, oye, vamos a ir a tal lado', pero yo: 'ahh, es que tengo que ir a nadar'. (...) igual antes como que me daba lo mismo, pero el año pasado ya no me daba lo mismo faltar a esas cosas, a las reuniones de amistades". E. 8.

"Aunque no quieran igual como que te excluyen un poco (...) después de clases 'vayamos a no sé, a tal parte', 'no, no puedo, porque tengo que ir a entrenar', y después los fines de semana, 'no, no puedo tengo que competir'. Al principio igual me seguían preguntando y todo, pero después ya ni me preguntaban, porque sabían que les diría que no. Igual como que después me da lata, porque llegan al colegio y dicen como que 'te acordái qué hicimos tal día', y como que tú nunca vái, entonces igual como que ellas se unen más y como que vái quedando afuera”. E. 5.

A pesar que todas las adolescentes relacionan aspectos con la permanencia y con dificultades asociadas a un riesgo de abandono, cabe señalar que las adolescentes, regulan y ponen en una balanza estos elementos, de diferentes maneras. Mientras algunas, ponen el foco en los aspectos positivos vinculados a la práctica, otras se centran en las dificultades de la práctica deportiva, vivenciándolo como un sacrificio.

"Igual esto es desgastante, pero te empieza a gustar tanto que se te olvida, olvidas que te cansa, y empiezas de nuevo al otro día. Es como si el día anterior no hubiese existido, que da lo mismo si estás cansada, igual como que a uno a veces le da lata... pero igual hay que pensar que esto igual te hace bien". E. 2 .
"No hay un sacrificio así en específico, no, porque yo lo disfruto". E. 1.

"Esto, o sea igual es un sacrificio, pero me gusta (...) Pero es el tiempo más que nada, porque, no sé, podría hacer otras cosas, pero prefiero venir para acá". E. 4 .

Cabe mencionar que aquellos casos que destacan los aspectos positivos de la práctica son adolescentes en que es posible apreciar una identidad centrada en el deporte o integradora; en cambio aquellos casos que enfatizan en las dificultades, coinciden con adolescentes con identidades no centradas en la práctica deportiva. Así, podríamos pensar que existe una relación entre el compromiso deportivo, vinculado a un deseo y decisión de seguir participando de la natación, relacionado con los diferentes aspectos que las adolescentes asocian con la permanencia deportiva, y el lugar que ocupa la práctica deportiva en la identidad.

\section{Discusión}

Dentro de las principales conclusiones del presente estudio se destacan las distintas posiciones que ocupa la práctica deportiva dentro de la identidad adolescente, además de cómo estas posiciones se relacionan con el compromiso deportivo. Así, respondiendo a la pregunta de investigación, podemos entender que las adolescentes nadadoras de nivel competitivo construyen su identidad de diferentes formas, pudiendo la identidad estar centrada en la práctica deportiva, no centrada en esta, o bien, ser una identidad integradora, que integre la práctica deportiva como parte de la identidad, así como otros elementos.

Además, estas diferentes posiciones de la práctica deportiva en la identidad se relacionan con los significados que las adolescentes asocian al compromiso deportivo, siendo las adolescentes con una identidad centrada en la práctica deportiva o aquellas que presentan una identidad integradora, quienes enfatizan los significados asociados al compromiso deportivo, en donde priman aspectos positivos asociados a la práctica deportiva. En cambio en las adolescentes que presentan una identidad no centrada en el deporte, priman aquellos significados asociados a dificultades y sacrificios de la práctica.

Es importante destacar que, independiente de la posición que ocupe la práctica deportiva en la construcción de identidad adolescente en la muestra estudiada, es posible reconocer en todas ellas un desarrollo de características adaptativas y acordes al periodo evolutivo que cursan. Sin embargo, es posible hipotetizar diferentes riesgos para la salud mental o para la permanencia en la práctica 
deportiva, dependiendo del lugar que ocupa el deporte en la identidad personal.

Así, podemos pensar que, en el caso de la identidad centrada en la práctica deportiva, constituida como la más viable en cuanto a la posibilidad de que las adolescentes puedan obtener significativos logros deportivos con proyección profesional, es también la que posee mayores riesgos de generar dificultades en el proceso de desarrollo adolescente, al existir una excesiva tendencia a considerar la práctica como único referente identitario, pudiendo descuidar otras áreas del desarrollo. Asimismo, ante situaciones vinculadas a mayores exigencias $o$ frustraciones, es posible que potencie la presencia de psicopatología, al estar las adolescentes fijadas en estructuras rígidas e inflexibles (Sepúlveda y Capella, 2012) o que esto facilite deseos de abandono definitivo de la práctica, lo que ha sido descrito en adultos, en la medida que una identidad muy centrada en lo deportivo podría implicar dificultades en las transiciones durante la carrera deportiva (Wylleman y Lavalle, 2004).

En cuanto a la identidad integradora, se distingue como un modo más adaptativo y flexible que el anterior, favoreciendo un desarrollo adecuado a la etapa evolutiva en que se encuentran las adolescentes, en equilibrio con la práctica deportiva. Sin embargo, podría presentar la dificultad de no potenciar una proyección de nivel competitivo con características profesionales, desviando los intereses hacia aspectos de otra índole.

Finalmente, en el caso de la identidad no centrada en la práctica deportiva, las diferencias más acentuadas entre este y los otros lugares que ocupa la práctica, radican en un mayor riesgo de abandono deportivo a nivel competitivo, aunque continúan dentro de lo que se considera un desarrollo adaptativo. El que estas adolescentes decidan abandonar la práctica podría ser consecuencia de que ni la pertenencia social ni identitaria se encuentra en el deporte y que se centran en las dificultades que implica su práctica.

En relación a esto, cabe señalar que en un seguimiento realizado a las adolescentes casi tres años después de su participación en la investigación (primer semestre de 2014), se apreció una vinculación entre el compromiso deportivo y el lugar que ocupaba la práctica deportiva en la identidad, y su efectiva permanencia o abandono deportivo. Así, las adolescentes que presentaban una identidad centrada en el deporte o integradora continuaban participando de la natación de manera competitiva, ya sea en competencias federadas nacionales (participantes 2, 7, 9) o competencias universitarias (participantes 1, 3, 5). En cambio, las adoles- centes en que se identificó una identidad no centrada en la práctica deportiva, no continúan practicando natación de manera competitiva (participantes 4, 6, 8, 10), haciendo abandono deportivo.

En relación a esto, otro elemento relevante de esta investigación fue identificar los aspectos que las adolescentes asociaron a la permanencia deportiva o a las dificultades de permanencia en esta. Dentro de los vinculados a la permanencia deportiva y a su compromiso de seguir practicando natación, señalan la importancia de la figura del entrenador, los vínculos con los compañeros de equipo, el disfrute y sentido de pertenencia. Por otro lado, los elementos asociados por las adolescentes a dificultades son la alta exigencia percibida, frustraciones, lesiones deportivas y relaciones interpersonales restringidas a la práctica deportiva. Estos elementos encontrados en la presente investigación coinciden con aquellos planteados por diversos autores a nivel internacional (Cudini, 2003; Fraser-Thomas, Coté y Deakin, 2008; Garcés, 1995; Guillén, Álvarez, García y Dieppa, 2007; Molinero, Salguero, Tuero, Álvarez y Márquez, 2006; Torregrosa et al., 2007).

Cabe destacar que uno de los factores por el que las jóvenes no deseaban mantenerse en la práctica deportiva es que les quitaba tiempo para compartir con sus amigos. Si bien esta situación no interferiría en el desarrollo normal de las adolescentes, ya que el interés por los círculos de amistades y distintas actividades es propia de la etapa adolescente y de vital importancia en la construcción identitaria (Sepúlveda, 2008), sí podría dificultar la permanencia en el deporte.

Esto coincide con lo planteado por autores como FraserThomas et al. (2008) que dan cuenta de la importancia de la participación de los amigos significativos en la práctica deportiva. De no ser así, y ante la presencia de vínculos cada vez más débiles dentro del equipo deportivo, es posible distinguir un menor compromiso con la actividad, interrumpiendo la práctica incluso de forma definitiva (Guillén et al., 2007).

De esta manera, y en relación a la importancia de la integración con los otros en el proceso de construcción la identidad (Sepúlveda, 2008), es relevante que parte importante de los vínculos afectivos seguros que construyen las adolescentes sea en el contexto deportivo.

En cuanto a los aportes de la presente investigación, además de dar a conocer la construcción de significados asociados a la práctica deportiva de nivel competitivo, que se vincularían a un mayor compromiso deportivo, se constituye también como un valioso aporte al plantear cómo el lugar que 
ocupa la práctica deportiva en la construcción de identidad adolescente también puede intervenir en un mayor o menor compromiso deportivo, gracias a la descripción cualitativa del cómo la práctica se integra en la vida de las adolescentes, en su visión de futuro, en su relación con los otros, etc.

Considerando todos estos aspectos es que el concepto de identidad deportiva, planteado como el grado en que las personas se identifiquen con el rol del deportista (Vázquez, 2008), es bastante restringido en la medida en que enfatiza solo en cuánto un individuo se pueda o no identificar con la práctica que realiza, centrándose exclusivamente en el desempeño deportivo de la persona.

A partir de la presente investigación, y desde el constructivismo evolutivo, el concepto de identidad deportiva puede ser considerablemente complementado, al considerar los principales postulados teóricos de este enfoque, que enfatizan en la importancia de la construcción de la identidad personal autónoma y solidaria como la meta central del desarrollo (Sepúlveda, 2008). De esta manera, la identidad personal se constituye en un concepto más integrador que el propuesto desde la psicología deportiva, ya que consideraría las distintas áreas del desarrollo y su relación entre sí y el medio, además de subrayar el modo en que las personas construyen y significan sus propias experiencias.

Desde esta visión se consideraría a las deportistas no solo a partir de su rol de competidoras, sino que también contemplando las tareas evolutivas de la adolescencia acorde a su desarrollo psicológico y de salud mental. De esta forma, al pensar en la permanencia deportiva, no solo habría que identificar la identidad deportiva, sino cómo esta confluye con otros aspectos del desarrollo, tal como se vio en esta investigación, como la relación con los pares, relación con el entrenador, proyectos académicos, etc.

Dentro de las proyecciones y futuras intervenciones, es importante considerar que el conocer los significados que las adolescentes asocian a la práctica deportiva de nivel competitivo, así como aquellos factores que incidirían en el compromiso deportivo, facilitaría la elaboración e implementación de programas de intervención relacionados no solo con la prevención del abandono deportivo adolescente, sino que también con fortalecer aquellos vínculos que funcionan como factores protectores.

Este tipo de intervenciones posicionaría al psicólogo en un rol de facilitador de un desarrollo evolutivo sano dentro de la práctica deportiva adolescente, no limitándose exclusivamente a intervenciones relacionadas con aspectos deportivo-competitivos, sino que incluyendo aspectos de la praxis clínica, con el objeto de lograr un desarrollo integral y adaptativo a la etapa evolutiva en que se encuentran. Así, se puede favorecer tanto la continuidad en la práctica deportiva de nivel competitivo, como el logro de las metas evolutivas propias de la adolescencia, como la identidad personal.

No obstante los aportes, cabe señalar limitaciones del presente estudio, que pueden ser subsanadas en futuras investigaciones. Dentro de esto, al ser la muestra de tamaño restringido, con pertenencia a un mismo club deportivo, que se realizaron solo análisis cualitativos y en base solamente a las perspectivas de las entrevistadas, esto puede haber sesgado los resultados y limitar la generalización de las conclusiones. Es factible, en futuras investigaciones, contrastar estos resultados con la utilización de metodología cuantitativa.

Además, es importante mencionar que, al momento de realizar el estudio, todas las adolescentes presentaban permanencia deportiva, por lo que para poder profundizar el vínculo entre identidad y compromiso deportivo, y su potencial relación con el abandono y permanencia deportiva, sería importante incorporar en futuros estudios la perspectiva de adolescentes desertoras de la práctica deportiva. También, cabe incorporar en futuros estudios a adolescentes varones, así como a competidores de diferentes deportes, para ampliar la comprensión de la identidad y el compromiso deportivo.

\section{Referencias}

Anderson, C., Masse, L., Zhang, H., Coleman, K. y Chang, S. (2009). Contribution of athletic identity to child and adolescent physical activity. American Journal of Preventive Medicine, 37(3), 220-226. doi:10.1016/j.amepre.2009.05.017

Andréu, J. (2000). Las técnicas de análisis de contenido: una revisión actualizada. Barcelona: Paidós.
Capella, C. (2011). Hacia narrativas de superación: el desafío para la psicoterapia con adolescentes de integrar la experiencia de agresión sexual a la identidad personal (Tesis doctoral inédita). Universidad de Chile, Santiago, Chile.

Capella, C. (2013). Una propuesta para el estudio de la identidad con aportes del análisis narrativo. Psicoperspectivas, 12(2), 117128. doi:10.5027/psicoperspectivas-vol13-issue2-fulltex-281 
Carbonell, S. (2008). Perfiles motivacionales de nadadores profesionales y nadadores amateur en Chile: estudio comparativo (Tesis de maestría inédita). Universidad Diego Portales, Santiago, Chile.

Cornejo, M., Mendoza, F. y Rojas, R. (2008). La investigación con relatos de vida: pistas y opciones del diseño metodológico. Psykhe, 17(1), 29-39. doi: 10.4067/S0718-22282008000100004

Cornejo, M. y Salas, N. (2011). Rigor y calidad metodológicos: un reto a la investigación social cualitativa. Psicoperpectivas, 10(2), 12-34. doi: 10.5027/psicoperspectivas-Vol10-Issue2fulltext-144

Cudini, M. (2003). Deserción en la práctica del voleibol femenino de Puerto General San Martín (Tesis de maestría inédita). Universidad Abierta Interamericana, Buenos Aires, Argentina.

Díez, C. y Hernández, J. (2006). ¿Acaso no hay diosas en el Olimpo? Práctica deportiva y sistema de género: apuntes de una investigación en marcha. En L. Cantarero, F. X. Medina y R. Sánchez (Coords.), Actualidad en el deporte: investigación $y$ aplicación (pp. 147-163). Donostia: Ankulegi Antropologia Elkartea.

Fraser-Thomas, J., Coté, J. y Deakin, J. (2008). Examining adolescent sport dropout and prolonged engagement from a developmental perspective. Journal of Applied Sport Psychology, 20(3), 318-333. doi: 10.1080/10413200802163549

Garcés, E. (1995). Burnout en niños y adolescentes: un nuevo síndrome en psicopatología infantil. Psicothema, 7(1), 33-40.

García, E., Gil, J. y Rodríguez, G. (1999). Metodología de la investigación cualitativa. Archidona: Ediciones Aljibe.

Guillén, F., Álvarez, M., García, S. y Dieppa, M. (2007). Motivos de participación deportiva en natación competitiva en niños y jóvenes. Cuadernos de Psicología del Deporte, 7(2), 59-74.

Guillén, F., Weis, G. y Navarro, M. (2005). Motivos de participación deportiva de niños brasileños atendiendo sus edades. Apunts, Pedagogía Deportiva, 80(19), 29-36.

Haro Navejas, F. (2007). Al asalto del Olimpo: deporte, identidad y género en la República Popular China. Estudios de Asia y África, 42(1), 51-75.

Molinero, O., Salguero, A., Tuero, C., Álvarez, E. y Márquez, S. (2006). Dropout reasons in young Spanish athletes: relationship to gender, type of sport and level of competition. Journal of Sport Behavior, 29(3), 255-270.

Organización Mundial de la Salud (OMS). (2011). Programa de salud del adolescente. Recuperado de http://www.who.int/ topics/adolescent_health/es/
Ruiz, L., Durán, J. y Arruza, J. (2007). Rendimiento experto y percepción de las actividades de entrenamiento en lanzadores de martillo españoles de alto rendimiento. Anales de Psicología, 23(2), 300-306.

Sepúlveda, G. (2006). Desarrollo psicológico en la edad juvenil: construcción de la identidad personal hacia la autonomía. En M. Valdivia y M. I. Condeza (Eds.), Psiquiatría del Adolescente (pp. 19-36). Santiago: Mediterráneo.

Sepúlveda, G. (2008). Perspectiva constructivista evolutiva en psicología clínica infanto-juvenil. En A. Kaulino y A. Stecher (Eds.), Cartografía de la Psicología contemporánea: pluralismo y modernidad (pp. 207-228). Santiago: LOM Ediciones.

Sepúlveda, G. y Capella, C. (2012). Desarrollo psicológico del escolar y sus trastornos: lo evolutivo y lo psicopatológico. En C. Almonte y E. Montt (Eds.), Psicopatología infantil y de la adolescencia (pp. 33-48). Santiago: Mediterráneo.

Sparkes, A. y Devis, J. (2007). Investigación narrativa y sus formas de análisis: una visión desde la educación física y el deporte. En W. Moreno Gómez (Ed.), Educación, cuerpo y ciudad: el cuerpo en las interacciones e instituciones sociales (pp. 43-68). Medellín: Funanbulos editores.

Sparkes, A. y Smith, B. (2001). Hombres, deporte, lesión en la médula espinal y problemas en la restauración del yo. Ágora para la Educación Física y el Deporte, 1, 37-48.

Torregrosa, M., Cruz, J., Sousa C., Viladrich, C., Villamarín, F., García-Mas, A. y Palou, P. (2007). La influencia de padres y madres en el compromiso deportivo de futbolistas jóvenes. Revista Latinoamericana de Psicología, 39(2) 227-237.

Vasalo, C. (2001). Competitividad en deportes infantiles. PubliCE. Standard. Recuperado de: http://g-se.com/es/psicologiadel-deporte/articulos/competitividad-en-deportesinfantiles-97

Vázquez, B. (Noviembre, 2008). ¿Cómo construimos nuestra identidad a través del deporte? Trabajo presentado en $5^{\circ}$ Jornadas de Igualdad: Practicando la igualdad a través del deporte. Galdakao, País Vasco, España. Recuperado de http:// menesianos.jimdo.com/app/download/4223645857/como_ construimos_identidad_deporte.pdf?t=1398867076

Wylleman, P. y Lavalle, D. (2004). A developmental perspective on transitions faced by athletes. En M. Weiss (Ed.), Developmental sport and exercise psychology: A lifespan perspective (pp. 507-527). Morgantown: Fitness Information Technology. Recuperado de http://drmichellecleere. com/wp-content/uploads/downloads/2013/01/LifeSpan_ Chap19_screen2.pdf 